\title{
Comparable neuroprotective effect of rapamycin against low and high rotenone concentrations in primary dopaminergic cell culture
}

\author{
Khaled Radad $^{1 *}$, Rudolf Moldzio ${ }^{2}$, Mubarak Al-Shraim ${ }^{3}$, Ahmed Al-Emam ${ }^{4}$, Wolf-Dieter Rausch ${ }^{2}$ \\ ${ }^{1}$ Department of Pathology, Faculty of Veterinary Medicine, Assiut University, Assiut 71526, Egypt. ${ }^{2}$ Institute of Medical Biochemistry, Department for \\ Biomedical Sciences, University of Veterinary Medicine, Veterinaerplatz 1, A-1210 Vienna, Austria. ${ }^{3}$ Department of Pathology, College of Medicine, King \\ Khalid University, Abha, Saudi Arabia. ${ }^{4}$ Department of Forensic Medicine and Toxicology, Faculty of Medicine, Mansoura University, Mansoura, Egypt.
}

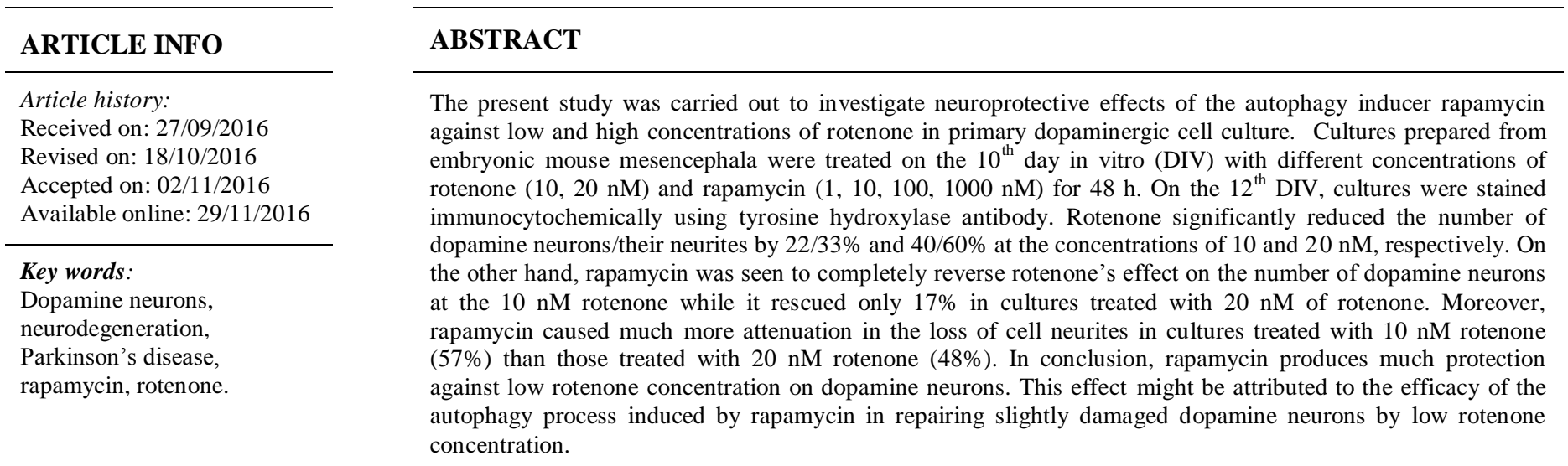

\section{INTRODUCTION}

Parkinson's disease (PD) is a chronic neurodegenerative disorder with a global prevalence of $1-3 \%$ in the population over 55 years (Fahn and Sulzer, 2004). The disease is characterized by preferential death of dopamine neurons in substantia nigra pars compacta (SNpc) and subsequent depletion of dopamine levels in the striatum (Li et al., 2015). This leads to the cardinal motor symptoms including resting tremors, bradykensia, muscle rigidity and postural instability. These motor signs may be accompanied by some non-motor symptoms such as constipation, urinary symptoms, sleep disorders and dementia

\footnotetext{
* Corresponding Author

Khaled Radad, Department of Pathology, Faculty of Veterinary

Medicine, Assiut University, Assiut 71526, Egypt.

Email: khaledradad@ hotmail.com
}

(Fahn, 2003). Although the etiology of PD is still elusive, two forms of the disease are recognized, genetic and sporadic forms. Genetic form represents approximately $5-10 \%$ of the cases and results from mutations in some gene loci including alphasynuclein, parkin, PINK, LRRK2 and some other genes (Singleton et al., 2013). Sporadic PD affects $90 \%$ of the cases and is thought to be associated with environmental exposures, most notably to pesticides (de Lau and Breteler, 2006; Lees et al., 2009). Liu et al., (2015) reported that there is a strongest association between pesticides and the incidence of PD. Rotenone is a naturally occurring insecticide that derived from the roots of Derris and Lanchorcarpus plant species. It is used as an active ingredient in hundreds of pesticide products worldwide (Betarbet et al., 2000). Upon exposure, rotenone as a hydrophobe easily crosses biological membranes and reaching mitochondria where it produces inhibition of complex I of respiratory chain (NADHdehydrogenase) (Worth et al., 2014). 
Since 2000 when Betarbet and her colleagues (Betarbet et al., 2000) used rotenone to reproduce pathological features characteristic of PD in rats, rotenone has been suggested to play an important role in the development of the disease (Brown et al., 2006). At present, levodopa therapy remains the most effective symptomatic treatment for PD patients (Singer, 2012). With time, levodopa therapy becomes inadequate in controlling motor fluctuation and dyskinesias (Worth, 2013). Moreover, levodopa undergoes auto-oxidation and forms reactive oxygen species (ROS) which could be toxic to surviving neurons (Lipski et al., 2011). Dopamine agonists are used for symptomatic treatment of early PD patients. They are beneficial over levodopa in minimizing the risk of the development of dyskinesias due to their longer half-life (Stathis et al., 2015).

Neuroprotective strategies that can protect dopamine neurons from progressive damage have received much attention in the last two decades. So far, just only preclinical advances have been proposed to rescue dopamine neurons in PD but none of them has been proved in clinical trials (More and Choi, 2015). Most recently, autophagy dysfunction is linked to several neurodegenerative diseases including PD (Hu et al., 2015). For instance, Dehay et al., (2010) reported that the number of autophagosomes increased and autolysosomes decreased in PD brains.

In accordance, our current study was carried out to investigate comparable neuroprotective effects of the autophagy inducer rapamycin against low and high rotenone concentrations in primary dopaminergic cell culture relevant to PD.

\section{MATERIALS AND METHODS}

\section{Preparation of primary mesencephalic cell culture}

All experimental procedures in the present study were done in accordance with the guidelines of the European Union Council (86/609/EU). Primary dopaminergic cell cultures were prepared from OFI/SPF embryos according to Radad et al., (2015). In brief, embryonic mouse mesencephala were dissected on the $14^{\text {th }}$ day of gestation and cut into small pieces in a drop of DPBS (Invitrogen, Germany), $2 \mathrm{ml}$ of $0.2 \%$ trypsin solution (Invitrogen, Germany) and $2 \mathrm{ml}$ of $0.02 \%$ DNase I solution (Roche, Germany) were added and the tissue was subsequently incubated in a water bath at $37^{\circ} \mathrm{C}$ for $7 \mathrm{~min}$. Then, $2 \mathrm{ml}$ of trypsin inhibitor $(0.125$ $\mathrm{mg} / \mathrm{ml}$ ) (Invitrogen, Germany) were added, the tissue was centrifuged at 100x $\mathrm{g}$ for $4 \mathrm{~min}$ and the supernatant was aspirated. The tissue pellet was triturated 2-3 times with a fire-polished Pasteur pipette, each time $0.02 \%$ DNase I (Invitrogen, Germany) was included in the medium. Dissociated cells were plated at a density of 257.000 cells $/ \mathrm{cm}^{2}$ in DMEM (Sigma, Germany) supplemented with $4 \mathrm{mM}$ glutamine, $10 \mathrm{mM}$ HEPES buffer, 30 $\mathrm{mM}$ glucose, $100 \mathrm{IU} / \mathrm{ml}$ penicillin, $0.1 \mathrm{mg} / \mathrm{ml}$ streptomycin and $10 \%$ heat-inactivated fetal calf serum (Sigma, Germany).

The medium was exchanged on the $1^{\text {st }}$ day in vitro (DIV) and on the $3^{\text {rd }}$ DIV. On the $5^{\text {th }}$ DIV half of the medium was replaced by serum-free DMEM containing $0.02 \mathrm{ml}$ of B-27/ml (Invitrogen, Germany) DMEM. Serum-free supplemented DMEM was used for feeding from the $6^{\text {th }}$ DIV and subsequently replaced every $2^{\text {nd }}$ day.

\section{Treatment of cultures with rapamycin}

A stock solution of $1 \mathrm{mM}$ of rapamycin (Invitrogen, USA) was prepared in dimethyl sulfoxide (DMSO). The solution was further diluted in DMEM to final concentrations. To investigate whether rapamycin alone affects the survival of dopamine neurons, cultures were treated with different concentrations of rapamycin $(1,10,100$ and $1000 \mathrm{nM})$ on the $10^{\text {th }}$ DIV for $48 \mathrm{~h}$.

\section{Treatment of cultures with rapamycin and rotenone}

In each treatment, a fresh stock solution $(1 \mu \mathrm{M})$ of rotenone (Sigma-Aldrich, Germany) was prepared in DMSO. The solution was further diluted in DMEM to final concentrations. To investigate the neurotoxic effect of rotenone, two cultures were separately treated with 10 and $20 \mathrm{nM}$ rotenone on the $10^{\text {th }}$ DIV for $48 \mathrm{~h}$. On the other hand, another two cultures were concomitantly treated with rapamycin $(1,10,100,1000 \mathrm{nM})$, and 10 and $20 \mathrm{nM}$ rotenone, respectively, on the $10^{\text {th }}$ DIV for $48 \mathrm{~h}$ to investigate the effect of rapamycin against rotenone neurotoxicity.

\section{Identification of dopaminergic neurons}

Dopaminergic neurons were identified immunocytochemically using anti-tyrosine hydroxylase (TH) antibody. On the $12^{\text {th }}$ DIV, cultures were rinsed carefully with phosphate buffered saline (PBS, $\mathrm{pH}$ 7.2) and fixed in $4 \%$ paraformaldehyde for $45 \mathrm{~min}$ at $4{ }^{\circ} \mathrm{C}$. After washing with $\mathrm{PBS}$, cells were permeabilized with $0.4 \%$ Triton X-100 for $30 \mathrm{~min}$ at room temperature. Cultures were washed 3 times with PBS and incubated with 5\% horse serum (Vectastain ABC Elite kit) for 90 min to block nonspecific binding sites. To determine the number of THir cells, cultures were sequentially incubated with anti-TH primary antibody overnight at $4^{\circ} \mathrm{C}$, biotinylated secondary antibody (Vectastain) and avidin-biotin-horseradish peroxidase complex (Vectastain) for $90 \mathrm{~min}$ at room temperature and washed with PBS between stages.

The reaction product was developed in a solution of diaminobenzidine $(1.4 \mathrm{mM})$ in PBS containing $3.3 \mathrm{mM}$ hydrogen peroxide $\left(\mathrm{H}_{2} \mathrm{O}_{2}\right)$ and stained cells were counted with a Nikon inverted microscope in 10 randomly selected fields per well at 10x magnification.

\section{Statistics}

Each experiment was run in triplicate with four wells in each treatment. Data were expressed as mean \pm standard error of mean (SEM). Comparisons were made using ANOVA and posthoc Duncan's test using IBM SPSS statistics 22 . P $<0.05$ was considered as statistically significant. 


\section{RESULTS}

\section{Effect of rapamycin on the survival of dopamine neurons}

Treatment of cultures with rapamycin alone $(1,10,100$, $1000 \mathrm{nM}$ ) on the $10^{\text {th }} \mathrm{DIV}$ for $48 \mathrm{~h}$ affected neither the survival (Figure 1) nor the morphology of dopamine neurons (data not shown).

\section{Effect of rotenone on dopamine neurons}

Treatment of cultures with rotenone significantly decreased the survival and reduced the neurites number of dopamine neurons, and altered their morphology. At $10 \mathrm{nM}$, rotenone decreased the survival of dopamine neurons and their neurite number by $22 \%$ and $33 \%$, respectively, compared to untreated control (Fig. 2,3), and slightly altered their morphology (Fig. 4). While at $20 \mathrm{nM}$, rotenone produced much more decrease in the survival and the neurite number of dopamine neurons by about $40 \%$ and $60 \%$, respectively, than the cultures treated with 10 nM (Fig. 2,3). Moreover, such concentration markedly altered the morphology of dopamine neurons in the form of dysmorphic and shortened neurites (Fig. 4).

\section{Effect of rapamycin against rotenone-treated dopamine neurons}

Co-treatment of cultures with rapamycin $(100 \mathrm{nM})$ and rotenone completely attenuated rotenone's effect on the number of dopamine neurons in the $10 \mathrm{nM}$ rotenone-treated cultures compared to rescuing only $17 \%$ of dopamine neurons in $20 \mathrm{nM}$ rotenone-treated cultures (Fig. 2). Rapamycin (100 nM) was also seen to produce much attenuation in the loss of cell neurites in cultures treated with $10 \mathrm{nM}$ rotenone $(57 \%)$ than those treated with $20 \mathrm{nM}$ rotenone (48\%) (Fig. 3). Moreover, rapamycin markedly improved the morphology of dopamine neurons in the form of increasing their neurite lengths (Fig. 4).

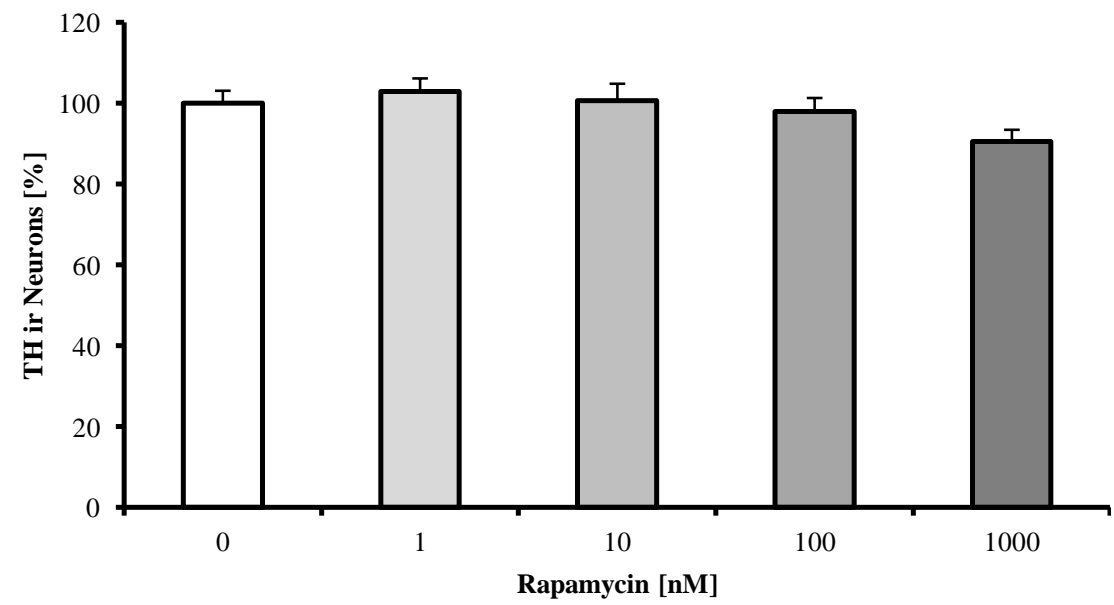

Fig. 1: Treatment of cultures with rapamycin on the $10^{\text {th }}$ DIV for $48 \mathrm{~h} .100 \%$ corresponds to the total number of dopamine neurons (the average number of dopamine neurons was 26 cells/field) after 12 DIV in untreated controls. Values represent the mean \pm SEM of three independent experiments with four wells in each treatment. In each well, dopamine neurons were counted in 10 randomly selected fields.

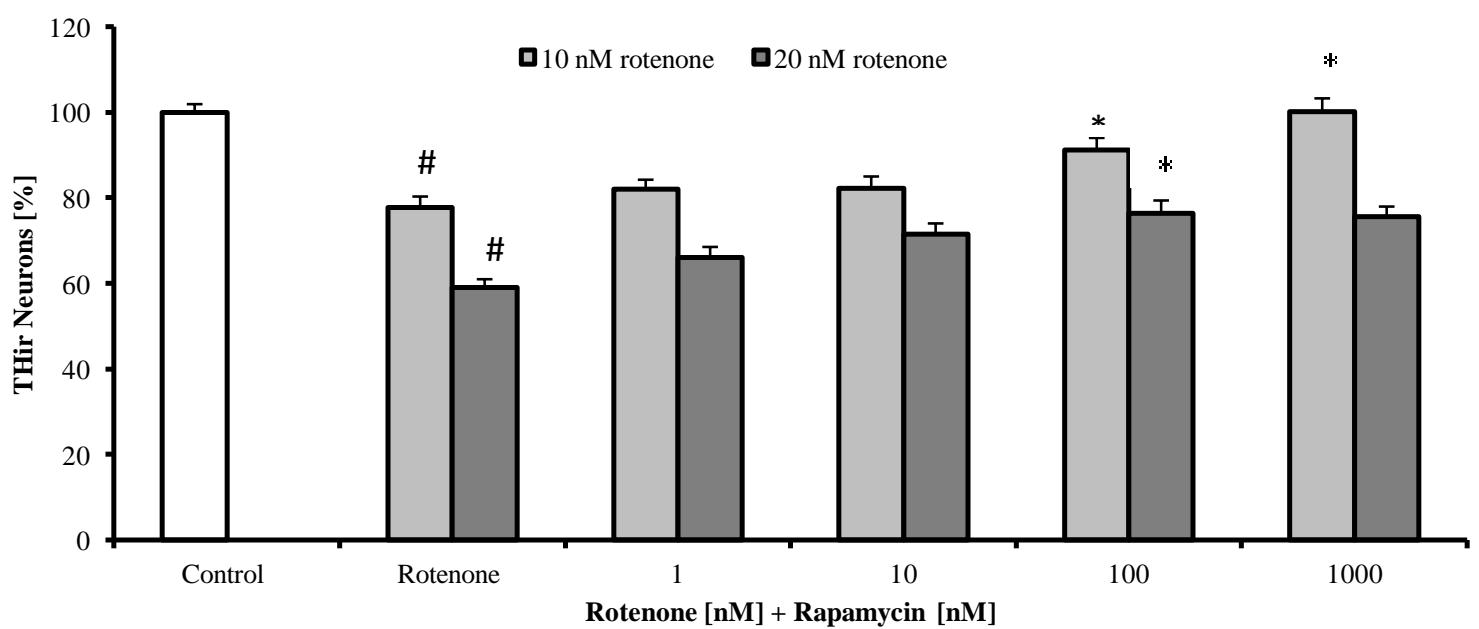

Fig. 2: Survival of dopamine neurons after concomitant treatment of cultures with rapamycin and rotenone on the $10^{\text {th }}$ DIV for $48 \mathrm{~h}$. $100 \%$ corresponds to the total number of dopamine neurons (the average number of dopamine neurons was 24 cells/field) after 12 DIV in untreated controls. Values represent the mean \pm SEM of three independent experiments with four wells in each treatment. In each well, dopamine neurons were counted in 10 randomly selected fields. $\left(\mathrm{p}^{\#, *}<\right.$ $0.001)$. 


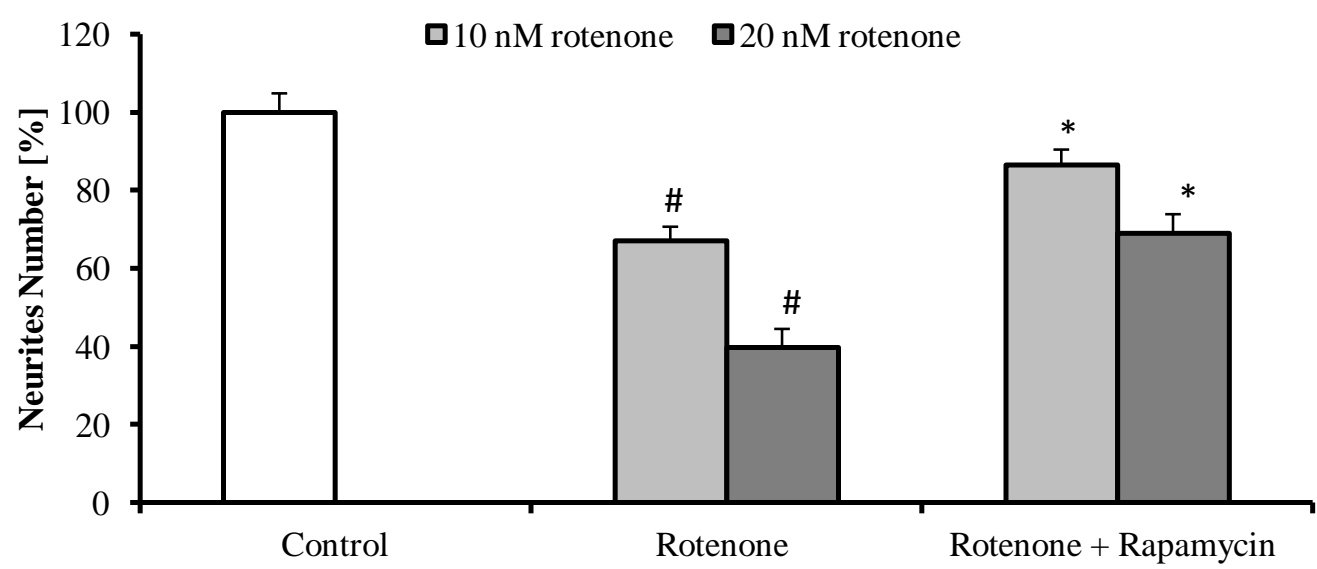

Fig. 3. The neurites number of dopamine neurons after concomitant treatment of cultures with rapamycin and rotenone on the $10^{\text {th }}$ DIV for $48 \mathrm{~h}$. $100 \%$ corresponds to the average number of neurites (4.12 neurites/cell) in untreated control cultures after 12 DIV. Values represent the mean \pm SEM of three independent experiments with four wells in each treatment. In each well, neurites number were counted for 5 dopamine neurons in 10 randomly selected fields (200 cell/experiment). $\left(\mathrm{p}^{\#, *}<0.001\right)$
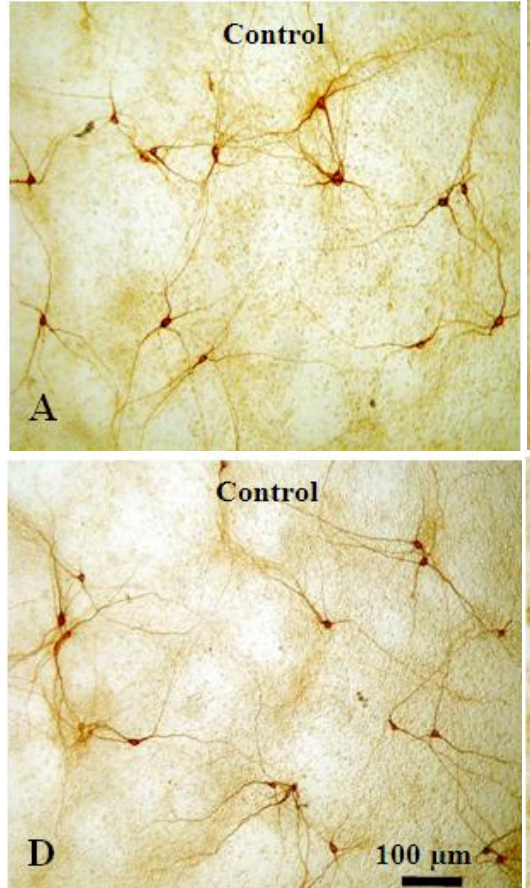
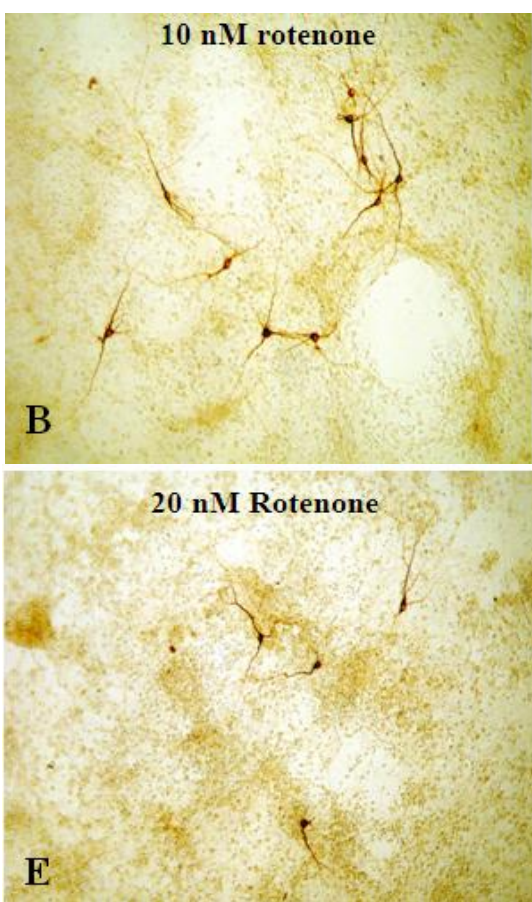
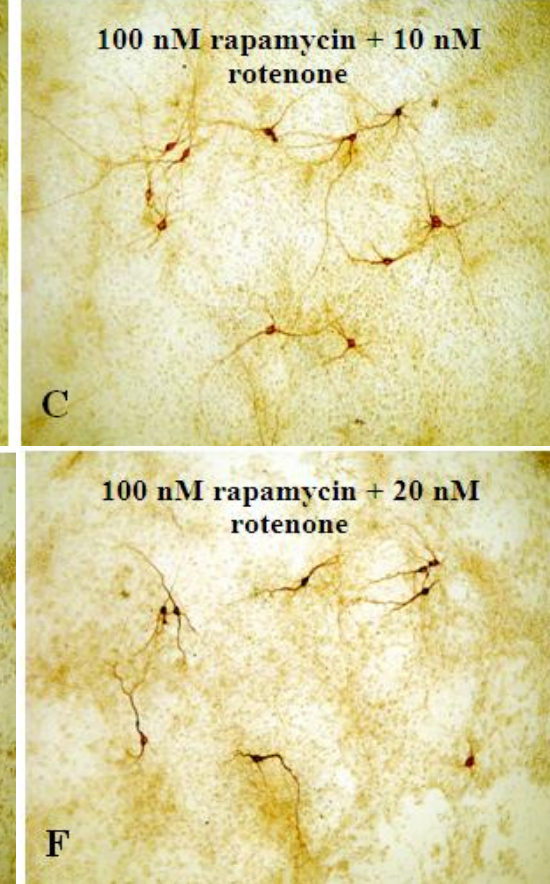

Fig. 4. Representative micrographs of dopamine neurons after 12 DIV. Untreated control cultures show dopamine neurons with long and branched neurites. Rotenone-treated cultures show much more morphological alteration in the $20 \mathrm{nM}$-treated cultures than $10 \mathrm{nM}$-treated ones. Co-treatment of cultures with rapamycin and rotenone markedly attenuated morphological alterations particularly in cultures treated with $20 \mathrm{nM}$ rotenone.

\section{DISCUSSION}

In the present study, dopaminergic cell culture was used to investigate the comparable neuroprotective effects of rapamycin against low and high rotenone concentrations relevant to PD. In which, neurotoxicity of the well-known and powerful complex I blocker rotenone to dopaminergic neurons was concentrationdependent. It decreased the survival of dopamine neurons and their neurites by $22 \%$ and $33 \%$, respectively at $10 \mathrm{nM}$ compared to $40 \%$ and $60 \%$ at $20 \mathrm{nM}$. Moreover, morphological alterations in the form of dysmorphic and shortened neurites were markedly seen at the concentration of $20 \mathrm{nM}$ more than at $10 \mathrm{nM}$ of rotenone.
Similarly, Radad et al., (2006) reported that rotenone challenged primary cultured dopamine neurons in a dose-dependent manner as it decreased survival of dopamine neurons by about $16 \%$ and $50 \%$ at the concentrations of 5 and $20 \mathrm{nM}$ of rotenone, respectively, compared to untreated controls. Moon et al., (2005) also observed that the cytotoxic damage after $24 \mathrm{~h}$ of rotenone treatment gradually increased from 5 to $20 \mathrm{nM}$ in neuron-enriched primary dopaminergic cultures. Similarly, rotenone decreased the viability of SH-SY5Y in a dose-dependent manner (Xiong et al., 2013). Majority of literature attributed rotenone's neurotoxicity to: (1) accumulation of $\alpha$-synuclein, (2) enhancement production of ROS and (3) activating caspase-3-mediated apoptosis. In this context, 
Radad et al., (2006) found that treatment of primary dopaminergic cell culture with $20 \mathrm{nM}$ of rotenone significantly decreased mitochondrial membrane potential $(\Delta \psi \mathrm{m})$ leading to increasing superoxide and ROS production, and enhancing apoptotic cell death. Xiong et al. (2013) reported that rotenone induced apoptotic cell death of SH-SY5Y through decreasing $\Delta \psi \mathrm{m}$ and increasing ROS. Satish Bollimpelli and Kondapi (2015) found that rotenone produced $\alpha$-synuclein aggregation, caspase- 3 activation and ROS production in primary cultured mature dopamine neurons. Against rotenone, the autophagy inducer rapamycin significantly protected dopamine neurons and their neurites. In $10 \mathrm{nM}$ rotenone-treated cultures, rapamycin completely blocked rotenone effect on the number of dopamine neurons and produced much more attenuation in neurite loss compared to rescuing only $17 \%$ of dopamine neurons and producing less attenuation in neurite loss at $20 \mathrm{nM}$ of rotenone. Rapamycin was similarly seen to protect dopaminergic neurons in some in in vitro and in vivo experimental models. For instance, Pan et al., (2009) reported that rapamycin alleviated rotenone-induced death in SH-SY5Y cells. Jiang et al., (2013) found that rapamycin protected against the loss of dopamine neurons in the 6-hydroxydopamine-induced rat model of PD. Neuroprotective effect of rapamycin was reported to be mediated by inactivation of the mammalian target of rapamycin (mTOR) leading to autophagy activation (Ding et al., 2015). In consistent, rapamycin was seen to increase the signal of Lysotracker Deep Red fluorescent dye in rotenone-treated primary dopaminergic cell culture leading to protection of $\Delta \psi \mathrm{m}$ and decreasing apoptotic cell death (Radad et al., 2015). Besides neuroprotection, our findings indicated that rapamycin's neuroprotective effect on dopamine neurons is conversely proportional to the damaging effects of rotenone. i.e. rapamycin neuroprotection depends on how much is the cellular damage? The effect which needs further clarification in future research, to determine to which point autophagy is protecting against neurodegeneration? In conclusion, rapamycin is much more effective against low rotenone concentration on dopamine neurons. This effect seems to depend on to what extent autophagy process by rapamycin could repair rotenone-induced damage on dopamine neurons.

\section{Financial support and sponsorship: Nil.}

Conflict of Interests: There are no conflicts of interest.

\section{REFERENCES}

Betarbet R, Sherer TB, MacKenzie G, Garcia-Osuna M, Panov AV, Greenamyre JT. Chronic systemic pesticide exposure reproduces features of Parkinson's disease. Nat Neurosci, 2000; 3:1301-1306.

Brown TP, Rumsby PC, Capleton AC, Rushton L, Levy LS. Pesticides and Parkinson's disease--is there a link? Environ Health Perspect, 2006; 114:156-164.

de Lau LM, Breteler MM. Epidemiology of Parkinson's disease. Lancet Neurol, 2006; 5:525-535.

Dehay B, Bové J, Rodríguez-Muela N, Perier C, Recasens A, Boya P, Vila M. Pathogenic lysosomal depletion in Parkinson's disease. J Neurosci, 2010; 30:12535-12544.

Ding K, Wang H, Wu Y, Zhang L, Xu J, Li T, Ding Y, Zhu L, He J. Rapamycin protects against apoptotic neuronal death and improves neurologic function after traumatic brain injury in mice via modulation of the mTOR-p53-Bax axis. J Surg Res, 2015; 194:239-247.

Fahn S, Sulzer D. Neurodegeneration and neuroprotection in Parkinson disease. NeuroRx, 2004; 1:139-154.

Fahn S. Description of Parkinson's disease as a clinical syndrome. Ann N Y Acad Sci, 2003; 991:1-14.

$\mathrm{Hu}$ Z, Yang B, Mo X, Xiao H. Mechanism and regulation of autophagy and its role in neuronal diseases. Mol Neurobiol, 2015; 52:1190-1209.

Jiang J, Jiang J, Zuo Y, Gu Z. Rapamycin protects the mitochondria against oxidative stress and apoptosis in a rat model of Parkinson's disease. Int J Mol Med, 2013; 31:825-832.

Lees AJ, Hardy J, Revesz T. Parkinson's disease. Lancet, 2009; 373:2055-2066

Li J, Li S, Zhang L, Ouyang L, Liu B. Deconvoluting the complexity of autophagy and Parkinson's disease for potential therapeutic purpose. Oncotarget, 2015; 6:40480 - 94045.

Lipski J, Nistico R, Berretta N, Guatteo E, Bernardi G, Mercuri NB. L-DOPA: a scapegoat for accelerated neurodegenerationin Parkinson's disease? Prog Neurobiol, 2011; 94:389-407.

Liu Y, Sun JD, Song LK, Li J, Chu SF, Yuan YH, Chen NH. Environment-contact administration of rotenone: A new rodent model of Parkinson's disease. Behav Brain Res, 2015; 294:149-161.

Moon Y, Lee KH, Park JH, Geum D, Kim K.Mitochondrial membrane depolarization and the selective death of dopaminergic neurons by rotenone: protective effect of coenzyme Q10. J Neurochem, 2005; 93:1199-1208.

More SV, Choi DK.Promising cannabinoid-based therapies for Parkinson's disease: motor symptoms to neuroprotection. Mol Neurodegener, 2015; 8:10:17.

Pan T, Rawal P, Wu Y, Xie W, Jankovic J, Le W. Rapamycin protects against rotenone-induced apoptosis through autophagy induction. Neuroscience, 2009; 164:541-551.

Radad K, Rausch WD, Gille G. Rotenone induces cell death in primary dopaminergic culture by increasing ROS production and inhibiting mitochondrial respiration. Neurochem Int, 2006; 49:379-386.

Radad K, Moldzio R, Rausch WD. Rapamycin protects dopaminergic neurons against rotenone-induced cell death in primary mesencephalic cell culture. Folia Neuropathol, 2015; 53:250-261.

Satish Bollimpelli SV, Kondapi AK. Differential sensitivity of immature and mature ventral mesencephalic neurons to rotenone induced neurotoxicity in vitro. Toxicol In Vitro, 2015; 30:545-551.

Singer C. Managing the patient with newly diagnosed Parkinson disease. Cleve Clin J Med, 2012; 9:3-7.

Singleton $\mathrm{AB}$, Farrer $\mathrm{MJ}$ and Bonifati V. The genetics of Parkinson's disease: progress and therapeutic implications. Movement Disorders, 2013; 2:814-823.

Stathis P, Konitsiotis S, Antonini A. Dopamine agonists early monotherapy for the delay of development of levodopa-induced dyskinesias. Expert Rev Neurother, 2015; 15:207-213.

Worth AJ, Basu SS, Snyder NW, Mesaros C, Blair IA. Inhibition of neuronal cell mitochondrial complex I with rotenone increases lipid $\beta$-oxidation, supporting acetyl-coenzyme A levels. J Biol Chem, 2014; 289:26895-26903.

Worth PF. How to treat Parkinson's disease in 2013. Clin Med, 2013; 13:93-96

Xiong N, Xiong J, Jia M, Liu L, Zhang X, Chen Z, Huang J, Zhang Z, Hou L, Luo Z, Ghoorah D, Lin Z, Wang T. The role of autophagy in Parkinson's disease: rotenone-based modeling. Behav Brain Funct, 2013; 13:1-12.

\section{How to cite this article:}

Radad K, Moldzio R, Al-Shraim M, Al-Emam A, Rausch WD Comparable neuroprotective effect of rapamycin against low and high rotenone concentrations in primary dopaminergic cell culture. J App Pharm Sci, 2016; 6 (11): 142-146. 